\title{
KIF6 719Arg Genetic Variant and Risk for Thoracic Aortic Dissection
}

Olga A. lakoubova, MD, PhD'1, Carmen H. Tong, MS'1, Joseph Catanese, MS'1, Charles M. Rowland, MS', May M. Luke, PhD, MBA ${ }^{1}$, Maryann Tranquilli, MD², John A. Elefteriades, MD ${ }^{2 *}$

${ }^{1}$ Quest Diagnostics Research and Development, San Juan Capistrano, California, USA

${ }^{2}$ Aortic Institute at Yale-New Haven Hospital, Yale University School of Medicine, New Haven, Connecticut, USA

\begin{abstract}
Background: Carriers of the 719Arg variant in KIF6, compared with noncarriers, have been reported to be at greater risk for coronary heart disease (CHD) in six prospective studies. Because CHD, thoracic aortic dissection, and nondissection thoracic aortic aneurysm share some risk factors and aspects of pathophysiology, we investigated whether carriers of the 719Arg variant also have greater odds of thoracic aortic dissection or nondissected thoracic aortic aneurysm than noncarriers.

Methods: We genotyped 140 thoracic aortic dissection cases, 497 nondissection thoracic aortic aneurysm cases, and $\mathbf{2 7 5}$ disease-free controls collected in the United States, Hungary, and Greece and investigated the association between KIF6 719Arg carrier status and thoracic aortic dissection, and between KIF6 719Arg carrier status and nondissection thoracic aortic aneurysm, using logistic regression models adjusted for age, sex, hypertension, smoking, and country.

Results: The odds of aortic dissection were two-fold greater in KIF6 719Arg carriers compared with noncarriers (odds ratio (OR) 2.14, 95\% confidence interval (CI) 1.18-3.9). To account for the potential of concomitant CHD to confound the association between the KIF6 719Arg and thoracic aortic dissection, we repeated the analysis after removing subjects with concomitant CHD; the estimates for association of KIF6 719Arg carrier status remained essentially the same (OR 2.04, 95\% Cl 1.11-3.77). In contrast, KIF6 719Arg carrier status was not associated with risk for nondissection thoracic aortic aneurysm.

Conclusions: We observed an association of the KIFG 719Arg genetic variant with thoracic aortic dissection
\end{abstract}

Published by Science International Corp. ISSN 2325-4637

Fax +12037853552

E-Mail: aorta@scienceinternational.org

http://aorta.scienceinternational.org
Accessible online at:

http://aorta.scienceinternational.org in this multicenter case-control study. This association may enhance our management of patients with thoracic aortic disease.

Copyright @ 2016 Science International Corp.

\section{Key Words}

Aneurysm • Aorta • Dissection • KIF6 • Biomarker

\section{Introduction}

Without surgical treatment, thoracic aortic dissection and thoracic aortic aneurysm can be lethal diseases [1, 2]; however, with elective surgical treatment, aneurysm patients have a near-normal prognosis [3] Thus, early diagnosis and frequent monitoring with advanced imaging are the keys to survival via timely elective surgery for patients with thoracic aortic aneurysm. Because thoracic aortic aneurysm is asymptomatic until aortic dissection or aneurysm rupture occurs, detection methods, including advanced imaging, must be applied to asymptomatic individuals. Acute aortic dissection sometimes occurs without substantial aorta enlargement [4]. Even when it has occurred clinically, aortic dissection can be initially overlooked in up to $40 \%$ of cases and can be very deadly in such scenarios [5]. The mortality rate of untreated dissections of the ascending aorta approaches $1 \%$ per hour during the first 48 hours [6]. Thus, a noninvasive marker that can

* Corresponding Author:

John A. Elefteriades, MD

Aortic Institute at Yale-New Haven Hospital

Yale University School of Medicine

789 Howard Ave, Clinic Building CB317, New Haven 06519, CT, USA

Phone: +1 203785 2551, Fax: +1 203785 3552, E-Mail: john.elefteriades@yale.edu 
identify individuals at greater risk for thoracic aortic dissection or nondissection aneurysm before clinical manifestations of the disease would represent a substantial advance in clinical care.

Because coronary heart disease (CHD), thoracic aortic dissection, and thoracic aortic aneurysm share risk factors and some aspects of underlying pathophysiology (vessel wall inflammation, common risk factors of hypertension, familial clustering, and smoking) $[7,8]$, a genetic variant associated with risk for CHD could be also associated with risk for thoracic aortic dissection or aneurysm.

It has recently been reported that carriers of the 719Arg variant in the kinesin-like protein 6 (encoded by KIF6), compared with noncarriers, were at greater risk for CHD in six prospective studies [9-15]. These studies included two large population-based studies (ARIC, Atherosclerosis Risk in Communities [9] and CHS, Cardiovascular Health Study [10]) and placebo arms of four landmark randomized clinical trials (CARE, Cholesterol and Recurrent Events [11]; WOSCOPS, West of Scotland Coronary Prevention Study [11]; PROSPER, PROspective Study of Pravastatin in the Elderly at Risk [12]; and WHS, Women's Health Study [13]). In contrast to these earlier trials, the association of KIF6 719Arg with CHD failed to replicate in the placebo arms of the two more recent randomized clinical trials such as JUPITER (Intervention Trial Evaluating Rosuvastatin) [14] and HPS (Heart Protection Study) [15]. However, some important differences between the earlier and more recent trials may account for inconsistency in the observed association in these studies. For example, patients enrolled in the latter trials had lower low-density lipoprotein-cholesterol (LDL-C) at the time of randomization (in JUPITER, due to enrollment criteria; in HPS, due to the run-in period, which included treatment of placebo arm patients with high-dose simvastatin therapy). The lower LDL-C values in these studies could have ameliorated the risk associated with the deleterious KIF6 719Arg variant [16]. This thesis is supported by a recent meta-regression analysis of 88,535 participants by Ference, which included KIF6 negative studies such as case-control studies of angiographically defined coronary artery disease [17] in the HPS and JUPITER trials. This meta-regression analysis concluded that the KIF6 719Arg variant significantly increases vulnerability of patients to the harmful effect of high LDL-C levels on the risk of CHD.

Given the common aspects of pathogenesis between CHD and thoracic aortic dissection and nondissection thoracic aortic aneurysm, in this casecontrol study we investigated whether carriers of the CHD risk variant KIF6 719Arg have greater odds for thoracic aortic dissection or nondissection thoracic aortic aneurysm compared with noncarriers.

\section{Methods}

\section{Study Subjects}

Study subjects included 140 patients with thoracic aortic dissection, 497 patients with nondissecting thoracic aortic aneurysm, and 275 disease-free control subjects collected in the United States (Yale University), Hungary (Semmelweis University), and Greece (Athens Medical Center and Evangelismos Hospital). Characteristics of cases and controls are presented in Table 1. Controls included randomly selected unaffected individuals from Greek and Hungarian study centers (nonpatient visitors and staff) $(n=206)$ and unaffected spouses of patients with thoracic aortic aneurysm or dissection recruited at Yale University $(n=69)$. The patients from the United States, Hungary, and Greece (and the respective controls) were analyzed together to increase statistical power, despite the possibility for inherent differences between geographic and ethnic regions. To account for geographic differences, risk estimates were adjusted for the participating center (country). Controls generally shared the same ethnic background, geographic location, and environmental milieu as cases. The physicians caring for the patients verified the aneurysm and/or dissection phenotype from their own radiographic and surgical records, and provided the demographic and clinical data on the patients and controls.

None of the patients had Marfan's disease or Ehlers-Danlos syndrome. In the dissection group $(n=140), 77(55 \%)$ were ascending dissection patients, and 63 (45\%) descending. Among the aneurysms ( $n=497), 97(19.5 \%)$ were familial, and $58(11.7 \%)$ had a bicuspid aortic valve. Among the dissections, 25 (17.9\%) were familial, and $3(2.1 \%)$ had bicuspid aortic valve. Among the aneurysms, 369 (74.2\%) were in the aortic root or ascending aorta, 104 (20.9\%) were in the descending aorta, and 24 (4.8\%) patients had an aneurysm in both locations.

All subjects provided written informed consent to participate in the study. Both the study and informed consent procedure were approved by the institutional review boards of the participating centers: the Yale University Human Investigation Committee, the Semmelweis University (Budapest, Hungary) Institutional Review Board, the Athens Hospital Center (Athens, Greece) Institutional Review Board, and the Evangelismos General Hospital (Athens, Greece) Institutional Review Board. 
Table 1. Characteristics of subjects with dissection and nondissection aneurysm and controls.

\begin{tabular}{|c|c|c|c|c|c|}
\hline Characteristic & $\begin{array}{c}\text { Dissection } \\
(n=140)\end{array}$ & $\begin{array}{c}\text { Nondissection } \\
\text { Aneurysm } \\
(n=497)\end{array}$ & $\begin{array}{c}\text { Control } \\
(n=275)\end{array}$ & $p$ Value* & $p$ Value $^{\#}$ \\
\hline Age, y & $63 \pm 13.1$ & $64 \pm 13.8$ & $57 \pm 15.2$ & 0.001 & $<0.0001$ \\
\hline Male, n (\%) & $85(60.7)$ & $344(69.2)$ & 107 (39.2) & $<0.0001$ & $<0.0001$ \\
\hline Smoking status, n (\%) & $69(49.3)$ & $185(37.3)$ & $91(35.7)$ & 0.01 & 0.69 \\
\hline Hypertension, $n$ (\%) & $113(81.3)$ & $352(71.0)$ & $98(38.0)$ & $<0.0001$ & $<0.0001$ \\
\hline Center, $n(\%)$ & & & & $<0.0001$ & $<0.0001$ \\
\hline United States (Yale) & $100(71.4)$ & $347(69.8)$ & $69(25.1)$ & & \\
\hline Hungary & $28(20.0)$ & $86(17.3)$ & $111(40.4)$ & & \\
\hline Greece & $12(8.6)$ & $64(12.9)$ & $95(34.5)$ & & \\
\hline
\end{tabular}

* Dissection cases vs. controls, \# Nondissection aneurysm cases vs. controls

Data presented as mean \pm standard deviation for age and as a number (\%) of subjects for other variables.

$P$ values are from Fisher exact tests, except those for age, which are from Wilcoxon rank sum tests.

\section{Dissection and Aortic Aneurism Assessment}

Patient demographics and phenotypes were ascertained from clinical records available at each enrolling site. Aneurysm and dissection were documented conclusively by echocardiography, computerized tomography (CT), magnetic resonance imaging, or operative findings.

\section{Genotyping and Laboratory Measurements}

Genotypes for individual DNA samples were determined by real-time kinetic polymerase chain reaction as described previously [11].

\section{Statistical Analysis}

Differences in traditional risk factors between thoracic aortic dissection cases and controls or between nondissection thoracic aortic aneurysm cases and controls were assessed by the Fisher exact test or Wilcoxon rank sum test for discrete and continuous characteristics, respectively. An exact test was used to assess deviation of genotype frequencies from Hardy-Weinberg expectations. The associations of KIF6 Trp719Arg genotype with dissection or nondissection aneurysm were assessed using logistic regression models without or with adjusting for traditional risk factors including age (at the index aneurysm diagnosis or dissection event for cases, at enrollment for controls), sex, current smoker (versus not) status, hypertension (defined as systolic blood pressure $>140$ $\mathrm{mm} \mathrm{Hg}$, diastolic blood pressure $>90 \mathrm{~mm} \mathrm{Hg}$, a physician's diagnosis of hypertension, or the use of antihypertensive medications), and participating center. The difference in frequency of KIF6 719Arg carrier status among participating centers, while controlling for thoracic aortic disease, was tested for statistical significance with the Cochran-Mantel-Haenszel statistic. Information on concomitant CHD for thoracic aortic dissection cases was available for samples collected in the United States (Yale University). All other probability values are two-sided, and 95\% confidence intervals (Cls) are presented. All statistical tests were two-sided and performed using SAS version 9 (SAS Institute Inc., Cary, NC, USA).

\section{Results}

\section{Subject Characteristics and Allele Frequencies}

The characteristics of cases and controls are presented in Table 1. Traditional risk factors such as older age, hypertension, and male sex were significantly more prevalent among cases. The smoking status differed between thoracic aortic dissection cases and controls but not between nondissection thoracic aortic aneurysm cases and controls.

Among controls, the genotype frequencies were 14.5\% for ArgArg, 41.8\% for ArgTrp, and 43.6\% for TrpTrp; overall, $56.4 \%$ of controls carried at least one copy of the KIF6 719Arg variant. The carrier frequen$c y$ in controls in this genetic study did not differ from that in other large Caucasian populations such as ARIC ( $52.6 \%, p=0.24$ for difference between studies). Since the majority of cases were from the United States (Yale University) and the majority of controls were from Hungary and Greece, there was a potential for confounding in this study if the Hungarian and Greek 
controls had lower KIF6 719Arg carrier frequency than the controls from the United States. Therefore, we compared KIF6 719Arg carrier frequency in Hungarian and Greek controls with that in the controls from the United States; we found that the carrier frequency in Hungarian (56.8\%) and Greek (58.9\%) controls was not lower than in U.S. controls (52.2\%). KIF6 719Arg carrier frequency did not differ by participating center $(p>0.5)$ after controlling for disease status (Table 2). In addition, we adjusted association results for participating centers. The genotype distribution of the KIF6 719Arg variant among controls did not deviate from Hardy-Weinberg equilibrium expectations $(p=0.15)$.

\section{Association of KIF6 719Arg with Dissection and Nondissection Aneurysm}

Carriers of the KIF6 719Arg variant, compared with noncarriers, had greater odds of thoracic aortic dissection (odds ratio (OR) $2.14,95 \% \mathrm{Cl} 1.18-3.90$ ) after adjusting for age, sex, hypertension, smoking status, and participating center (Table 3 ).

Because concomitant CHD could potentially confound the observed association between the KIF6 719Arg variant and thoracic aortic dissection, we reanalyzed the data after excluding cases with known CHD. The magnitude of association between carrier status and thoracic aortic dissection remained largely unchanged after excluding those with CHD (adjusted OR 2.04, 95\% Cl 1.11-3.77; Table 3).

In contrast to thoracic aortic dissection, nondissection thoracic aortic aneurysm was not significantly associated with KIF6 719Arg carrier status, compared with noncarriers: adjusted OR for KIF6 719Arg carriers was $0.94,95 \% \mathrm{Cl}$ 0.64-1.38 (Table 4). Although KIF6 719Arg carriers, compared with noncarriers, were at greater risk for descending nondissection aneurysm (unadjusted OR 2.07, 95\% Cl 1.26-3.40), the association was no longer significant after adjusting for potential confounders (OR 1.73, 95\% Cl 0.91-3.29; Table 5).

\section{Discussion}

We found that the KIF6 719Arg variant, which has previously been associated with CHD in six prospective studies, is also associated with thoracic aortic

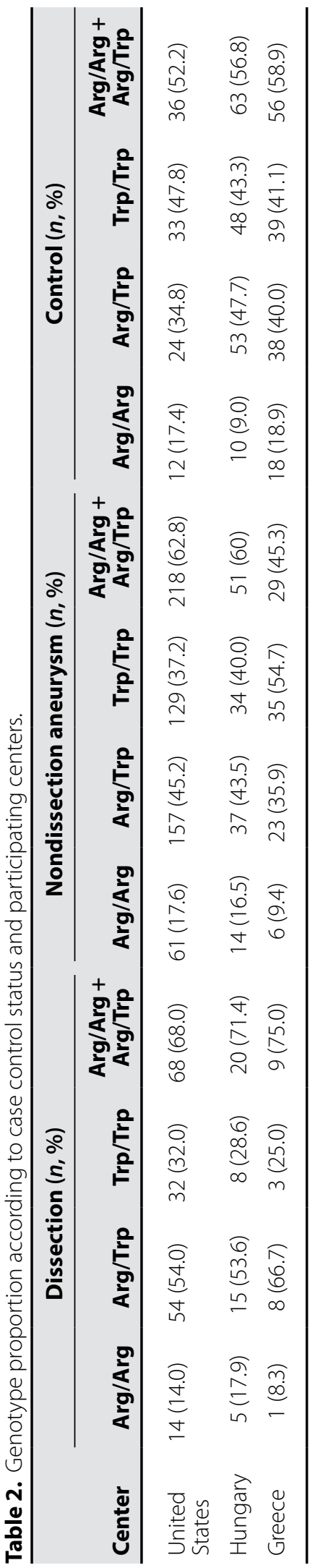


Table 3. Association of KIF6Trp719Arg with thoracic aortic dissection.

\begin{tabular}{|c|c|c|c|c|c|c|c|c|}
\hline \multirow[b]{2}{*}{ Genotype } & \multirow[b]{2}{*}{ Case } & \multirow[b]{2}{*}{ Control } & \multicolumn{3}{|c|}{ Unadjusted } & \multicolumn{3}{|c|}{ Adjusted* } \\
\hline & & & OR & $95 \% \mathrm{Cl}$ & $p$ Value & OR & $95 \% \mathrm{Cl}$ & $p$ Value \\
\hline \multicolumn{9}{|l|}{ Including CHD } \\
\hline Arg/Arg+Arg/Trp Arg/Trp Arg/Trp & 97 & 155 & 1.75 & $1.14-2.69$ & 0.0112 & 2.14 & $1.18-3.90$ & 0.013 \\
\hline $\mathrm{Arg} / \mathrm{Trp}$ & 77 & 115 & 1.87 & $1.19-2.94$ & 0.0068 & 2.39 & $1.27-4.48$ & 0.0066 \\
\hline Arg/Arg & 20 & 40 & 1.40 & $0.74-2.65$ & 0.3077 & 1.40 & $0.56-3.50$ & 0.4692 \\
\hline $\operatorname{Trp} / \operatorname{Trp}$ & 43 & 120 & ref & & & ref & & \\
\hline \multicolumn{9}{|l|}{ Without CHD } \\
\hline Arg/Arg + Arg/Trp & 81 & 155 & 1.70 & $1.07-2.67$ & 0.0234 & 2.04 & $1.11-3.77$ & 0.0224 \\
\hline $\mathrm{Arg} / \mathrm{Trp}$ & 63 & 115 & 1.78 & $1.10-2.87$ & 0.0189 & 2.23 & $1.17-4.23$ & 0.0144 \\
\hline Arg/Arg & 18 & 40 & 1.46 & $0.75-2.84$ & 0.2670 & 1.49 & $0.59-3.72$ & 0.3985 \\
\hline Trp/Trp & 37 & 120 & ref & & & ref & & \\
\hline
\end{tabular}

*Adjusted for sex, age, smoking (current versus noncurrent), country, and hypertension.

$\mathrm{CHD}=$ congestive heart disease; $\mathrm{Cl}=$ confidence interval; $\mathrm{OR}=$ odds ratio; ref $=$ reference.

Table 4. Association of KIF6Trp719Arg with nondissection aneurysm.

\begin{tabular}{|c|c|c|c|c|c|c|c|c|}
\hline \multirow[b]{2}{*}{ Genotype } & \multirow[b]{2}{*}{ Case } & \multirow[b]{2}{*}{ Control } & \multicolumn{3}{|c|}{ Unadjusted } & \multicolumn{3}{|c|}{ Adjusted* } \\
\hline & & & OR & $95 \% \mathrm{Cl}$ & $p$ Value & OR & $95 \% \mathrm{Cl}$ & $p$ Value \\
\hline Arg/Arg + Arg/Trp & 298 & 155 & 1.17 & $0.87-1.57$ & 0.3154 & 0.94 & $0.64-1.38$ & 0.7622 \\
\hline Arg/Trp & 217 & 115 & 1.14 & $0.83-1.58$ & 0.4114 & 0.96 & $0.64-1.44$ & 0.8267 \\
\hline Arg/Arg & 81 & 40 & 1.23 & $0.79-1.91$ & 0.3632 & 0.90 & $0.51-1.61$ & 0.7296 \\
\hline $\operatorname{Trp} / \operatorname{Trp}$ & 198 & 120 & ref & & & ref & & \\
\hline
\end{tabular}

*Adjusted for sex, age, smoking (current versus noncurrent), hypertension, and participating center.

$\mathrm{Cl}=$ confidence interval; $\mathrm{OR}=$ odds ratio; ref = reference.

Table 5. Association of KIF6 Trp719Arg with descending and ascending nondissection aneurysm.

\begin{tabular}{|c|c|c|c|c|c|c|c|c|}
\hline \multirow[b]{2}{*}{ Genotype } & \multirow[b]{2}{*}{ Case } & \multirow[b]{2}{*}{ Control } & \multicolumn{3}{|c|}{ Unadjusted } & \multicolumn{3}{|c|}{ Adjusted* } \\
\hline & & & OR & $95 \% \mathrm{Cl}$ & $p$ Value & OR & $95 \% \mathrm{Cl}$ & $p$ Value \\
\hline \multicolumn{9}{|l|}{ Descending } \\
\hline Arg/Arg+Arg/Trp Arg/Trp Arg/Trp & 75 & 155 & 2.07 & $1.26-3.40$ & 0.0039 & 1.73 & $0.91-3.29$ & 0.0965 \\
\hline Arg/Trp & 53 & 115 & 1.98 & $1.17-3.34$ & 0.0110 & 1.71 & $0.87-3.37$ & 0.1215 \\
\hline Arg/Arg & 22 & 40 & 2.36 & $1.21-4.58$ & 0.0113 & 1.78 & $0.73-4.33$ & 0.2057 \\
\hline $\operatorname{Trp} / \operatorname{Trp}$ & 28 & 120 & ref & & & ref & & \\
\hline \multicolumn{9}{|l|}{ Ascending } \\
\hline $\operatorname{Arg} / \operatorname{Arg}+\operatorname{Arg} / \operatorname{Trp}$ & 207 & 155 & 1.00 & $0.73-1.37$ & 0.9920 & 0.86 & $0.57-1.28$ & 0.4491 \\
\hline Arg/Trp & 153 & 115 & 1.00 & $0.71-1.40$ & 1.0 & 0.89 & $0.58-1.36$ & 0.5755 \\
\hline Arg/Arg & 54 & 40 & 1.01 & $0.63-1.62$ & 1.0 & 0.77 & $0.41-1.43$ & 0.4065 \\
\hline Trp/Trp & 160 & 120 & ref & & & ref & & \\
\hline
\end{tabular}

*Adjusted for sex, age, smoking (current versus noncurrent), hypertension and participating center.

$\mathrm{Cl}=$ confidence interval; $\mathrm{OR}=$ odds ratio; ref = reference. 
dissection. The ORs approaching 2.00 are quite substantial for association of a single genetic polymorphism with a complex clinical disease. The effect size for this association remained essentially unchanged after adjusting for sex, age, smoking, participating country, and hypertension. This association of the KIF6 719Arg variant with thoracic aortic dissection was not confounded by concomitant CHD, since the effect size was not attenuated and remained statistically significant after removing cases of the thoracic aortic dissection with CHD from the analysis.

The extension of a genetic association from CHD to thoracic aortic disease perhaps is not surprising given that both diseases share risk factors and some aspects of underlying pathophysiology. Another example of such extension is the genetic variant on chromosome 9p21 that has been reported to be associated with both CHD and abdominal aortic aneurysms, as well as intracranial aneurysms [18]. In contrast to the 9p21 genetic variant, KIF6 719Arg was associated with thoracic aortic dissection but not with nondissection thoracic aortic aneurysm. Although we have observed an association with descending, but not ascending, aortic aneurysm in the unadjusted model, effect size was attenuated and became insignificant after adjustment for sex, age, smoking, participating center, and hypertension. Interestingly, in a recent study by LeMaire, investigators observed larger effect size for the association of the genetic polymorphisms in the FBN1 gene with thoracic aortic dissection than for association with nondissection thoracic aortic aneurysm [19].

The KIF6 protein is a member of the kinesin superfamily of proteins that mediate the intracellular transport of organelles, protein complexes, and messenger ribonucleic acids [20]. Typical kinesins are homodimeric molecules consisting of two N-terminal domains ("heads") that move along microtubules and C-terminal domains ("tails") that directly interact with the transported cargos or indirectly through adapter molecules. The KIF6 polymorphism replaces a nonpolar residue (Trp) with a basic residue (Arg) near the putative cargo-binding tail domain; thus, it has the potential to alter the affinity for the cargo molecules or possibly to modulate motor activity of the KIF6 protein. These cargos, which have yet to be identified, may play a role in modulating cardiovascular risk and the statin response. In addition, KIF9, the closest homolog of KIF6, interacts with a member of the small guanosine triphosphatase family that promotes endothelial cell sprouting and cytoskeleton reorganization. A similar role for KIF6 in endothelial cell growth and function could provide a link between KIF6 and CHD pathogenesis. KIF6 ribonucleic acid has been shown to be expressed in coronary arteries (see data deposited by King et al [21]), and KIF6 expression is reportedly higher in healthy homozygous carriers of the chromosome 9p21 CHD and aortic dissection risk allele than in noncarriers of the 9p21 risk allele. In terms of any potential mechanisms for predisposition to aortic dissection, these are currently unknown.

This study had some limitations. This genetic study had a case-control design and therefore the deaths from thoracic aortic dissection or nondissection aneurysm rupture were not included in the analysis. The impact of absent lethal cases on the analysis can be substantial [16]; for example, a presumptive preferential mortality of Arg/ Arg homozygotes in addition to insufficient power might explain the smaller effect size for association of Arg/Arg genotype with nonlethal thoracic aortic dissection than the association of Arg/Trp heterozygotes. Because there were only a few non-Caucasian participants in this study, we analyzed only Caucasians; therefore, the association of the KIF6 719Arg with thoracic aortic dissection should be further investigated in large replication studies that would include additional ethnic groups. Also, due to the use of spousal controls in the U.S. cohorts, males and females predominated the patient and control groups, respectively. This was addressed by taking potential confounders into account in the adjusted analysis.

Also, there are differences in the underlying characteristics of the aortic aneurysm, aortic dissection, and control groups. While male sex, smoking, and hypertension (Table 1) can fairly be expected to predominate in cardiovascular disease, we do not have detailed information on other risk factors for our control group patients. Differences in underlying group characteristics may have introduced bias in our findings. Also, while inclusion of three geographic patient sources (United States, Hungary, Greece) enhances the generalizability of our findings, an imbalance between origin of patients and controls (most patients from United States, most controls from abroad) may introduce its own bias, although we adjusted the risk estimates for geographic location of the participating clinical center 
(country).

We feel this study raises the possibility that KIF6 variant status may serve as a predictor for likelihood of aortic dissection. However, there are several methodologic shortcomings of this initial examination of this topic. Additional work is clearly needed, with more ideal group matching, deeper background information on controls, and greater patient numbers. If our findings are borne out in subsequent prospective studies, assessing KIF6 status could reasonably be added to our armamentarium for predicting aortic dissection risk in patients with thoracic aortic aneurysm.

\section{Conclusion}

In this multicenter case-control study, the KIF6 719Arg genetic variant was associated with thoracic aortic dissection but not thoracic aortic aneurysm. If the association of the KIF6 719Arg variant with thoracic aortic dissection is further confirmed, this variant could be useful in assessing thoracic aortic dissection risk.

\section{Conflict of Interest}

The authors have no conflict of interest relevant to this publication.

\section{Comment on this Article or Ask a Question}

\section{References}

1. Elefteriades JA. Beating a silent killer. Sci Am. 2005;293:64-71. DOI: 10.1038/scientificamerican0805-64

2. Elefteriades JA, Farkas EA. Thoracic aortic aneurysms: clinically pertinent controversies and uncertainties. J Am Coll Cardiol. 2010;55:841-857. DOI: 10.1016/j. jacc.2009.08.084

3. Mok S, Ma W, Ahmed M, Charilaou P, Peterss $S$, Chou AS, et al. Twenty-five-year outcome of composite graft aortic root replacement: Near "curative" impact on aortic root disease. $52^{\text {nd }}$ Annual Meeting of Society of Thoracic Surgeons. Phoenix, AZ. Jan 23-27, 2016.

4. Ziganshin BA, Bailey $A E$, Coons $C$, Dykas $D$, Charilaou $\mathrm{P}$, Tanriverdi LH, et al. Routine genetic testing for thoracic aortic aneurysm and dissection in a clinical setting. Ann Thorac Surg. 2015;100:1604-1611. DOI: 10.1016/j.athoracsur.2015.04.106

5. Elefteriades JA, Barrett PW, KopfGS. Litigation in nontraumatic aortic diseases--a tempest in the malpractice maelstrom. Cardiology. 2008;109:263-272. DOI: 10.1159/000107790

6. Goldfinder JZ, Halperin JL, Marin ML, Stewart AS, Eagle KA, Fuster V. Thoracic aortic aneurysm and dissection. J Am Coll Cardiol. 2014;64:1725-1739. DOI: 10.1016/j. jacc.2014.08.025

7. Kahn RA, Stone ME, Moskowitz DM. Anesthetic consideration for descending thoracic aortic aneurysm repair. Sem Cardiothor Vasc Anesth. 2007;11:205-223. DOI: $10.1177 / 1089253207306098$

8. Ho SY, Sheppard MN. The aorta: embryology, anatomy, and pathology. In: Boudoulas
$\mathrm{H}$ and Stefanadis C (Eds.). The aorta: structure, function, dysfunction, and diseases. New York: Informa; 2009, p. 6-21.

9. Morrison AC, Bare LA, Chambless LE, Ellis SG, Malloy M, Kane JP, et al. Prediction of coronary heart disease risk using a genetic risk score: the Atherosclerosis Risk in Communities study. Am J Epidemiol. 2007;166:28-35. DOI: 10.1093/aje/kwm060

10. Schiffman D, O'MEara ES, Bare LA, Rowland CM, Louie JZ, Arellano AR, et al. Association of gene variants with incident myocardial infarction in the Cardiovascular Health Study. Arterioscler Thromb Vasc Biol. 2008;28:173-179. DOI: 10.1161/ATVBAHA.107.153981

11. lakoubova $\mathrm{OA}$, Tong $\mathrm{CH}$, Rowland $\mathrm{CM}$, Kirchgessner TG, Young BA, Arellano $A R$, et al. Association of the Trp719Arg polymorphism in Kinesin-like protein 6 with myocardial infarction and coronary heart disease in 2 prospective trials: the CARE and WOSCOPS Trials. J Am Coll Cardiol. 2008;51:435-443. DOI: 10.1016/j. jacc.2007.05.057

12. lakoubova $\mathrm{OA}$, Robertson $\mathrm{M}$, Tong $\mathrm{CH}$, Rowland CM, Catanese JJ, Blauw GJ, et al. KIF6 Trp719Arg polymorphism and the effect of statin therapy in elderly patients: results from the PROSPER study. Eur J Cardiovasc Prev Rehabil. 2010;17:455-461. DOI: 10.1097/ HJR.0b013e328336a0dd

13. Schiffiman D, Chasman DI, Zee RY, lakoubova OA, Louie JZ, Devlin JJ, et al. A kinesin family member 6 variant is associated with coronary heart disease in the Women's Health Study. J Am Coll Cardiol. 2008;51:444-448. DOI: 10.1016/j. jacc.2007.09.044

14. Ridker PM, MacFadyen JG, Glynn RJ, Chasman DI. Kinesin-like protein 6 (KIF6) polymorphism and the efficacy of rosuvastatin in primary prevention. Circ Cardiovasc Genet. 2011;4:312-317. DOI: 10.1161/ CIRCGENETICS.110.959353

15. Hopewell JC, Parish S, Clarke R, Armitage J, Bowman L, Hager J, et al. No impact of KIF6 genotype on vascular risk and statin response among 18,348 randomized patients in the heart protection study. J Am Coll Cardiol. 2011;50:2000-2007. DOI: 10.1016/j.jacc.2011.02.015

16. Williams P, Pendyala L, Superko R. Survival bias and drug interaction can attenuate cross-sectional case-control comparisons of genes with health outcomes. An example of the kinesin-like protein 6 (KIF6) Trp719Arg polymorphism and coronaryheart disease. BMC Med Genet. 2011;12:4250. DOI: $10.1186 / 1471-2350-12-42$

17. Ference BA, YooW, Flack JM, Clarke M. A common KIF6 polymorphism increases vulnerability to low-density lipoprotein cholesterol: two meta-analyses and a meta-regression analysis. PLoS One. 2011;6:e28834. DOI: 10.1371/journal.pone.0028834

18. Helgadottir A, Thorensson G, Magnusson $\mathrm{KP}$, Grétarsdottir S, Steinthorsdottir $\mathrm{V}$, Manolescu A, et al. The same sequence variant of 9p21 associates with myocardial infarction, abdominal aortic aneurysm and intracranial aneurysm. Nat Genet. 2008;40:217-224. DOI: 10.1038/ng.72 
19. Lemaire S, McDonald ML, Guo DC, Russell L, Miller CC 3rd, Johnson RJ, et al. Genome-wide association study identifies a susceptibility locus for thoracic aortic aneurysms and dissections spanning FBN1 at 15q21.1. Nat Genet. 2011;43:996-1000. DOI: $10.1038 /$ ng.934

20. Li Y, lakoubova OA, Shiffman D, Devlin J, Forrester JS, Superko HR. KIF6 polymorphism as a predictor of risk of coronary events and of clinical event reduction by statin therapy. Am J Cardiol. 2010;106: 994-998. DOI: 10.1016/j.amjcard.2010. 05.033
21. King JY, Ferrara R, Tabibiazar R, Spin JM, Chen MM, Kuchinsky A, et al. Pathway analysis of coronary atherosclerosis. Physiol Genomics. 2005;23:103-118. DOI: 10.1152/ physiolgenomics.00101.2005
Cite this article as: lakoubova OA, Tong $\mathrm{CH}$, Catanese J, Rowland CM, Luke MM, Tranquilli M, Elefteriades JA. KIF6 719Arg Genetic Variant and Risk for Thoracic Aortic Dissection. AORTA (Stamford). 2016;4(3):83-90. DOI: http://dx.doi. org/10.12945/j.aorta.2016.16.003 